\section{MicroRNAs in angiogenesis}

During sprouting angiogenesis, vascular endothelial growth factor (VEGF) activates filopodia formation and the directional migration of an endothelial cell, the tip cell, which takes the lead in forming the new vessel. The tip cell takes with it endothelial trailing stalk cells, which do not make filopodia and form the core of the new vessels. This process also requires inhibition of tip cell behaviour in stalk cells through crosstalk between VEGF and Notch signalling. Lawson and colleagues have delineated a microRNA cascade that modulates tip cell behaviour in zebrafish (Dev. Cell 22, 418-429; 2012).

Using deep sequencing, the authors identify miR-221 as a microRNA specifically induced in endothelial cells at the time of sprouting. They show that following VEGF-C receptor Flt4 loss of function, sprouting angiogenesis is inhibited in embryos injected with miR-221 morpholinos. They find that miR-221 knockdown impairs the migration and proliferation of tip cells, whereas endothelial cells overexpressing miR221 drive vessel sprouting, resembling Delta-4 (an endothelial Notch ligand) loss-of-function phenotypes. They further show that the excessive sprouting associated with Delta-4 loss of function is suppressed by miR-221 deficiency, and that miR-221 expression is inhibited by Notch signalling. Mechanistically, they find two targets of miR-221 that influence tip cell behaviour: the Cdk inhibitor p27, and the p85 $\alpha$ regulatory subunit of the PI(3)K (phosphatidylinositol-3kinase) complex. Overexpression of both targets negatively influences tip cell behaviour, whereas reducing their levels provides partial rescue of miR-221-associated angiogenic phenotypes. Thus, miR-221 provides a further level of regulation for tip and stalk cell behaviour to ensure correct sprouting angiogenesis. NLB

\section{Sec-rets of COPII coat formation}

Secretory proteins exit the endoplasmic reticulum in COPII-coated vesicles. Sec13 and Sec31 form the outer layer of the COPII coat and are thought to drive membrane curvature. Miller and colleagues present evidence that yeast $\mathrm{Sec} 13$ provides rigidity to the COPII coat (Science http://doi.org/hq7; 2012).

Sec13 has many distinct biological functions, but is dispensable in bypass-of-sec-thirteen (bst)mutant genetic backgrounds. The authors used a synthetic genetic array to uncover new BST genes and identified two predominant groups: genes involved in glycosylphosphatidylinositol (GPI) anchor remodelling, and genes required for exporting GPI-anchored proteins from the endoplasmic reticulum. As GPI-anchored proteins are asymmetrically distributed, and thus probably alter local membrane topology, these screen results indicate that $\operatorname{Sec} 13$ has a role in regulating membrane dynamics to accommodate these cargo proteins.

The authors went on to show that Sec31 could form vesicles in vitro in the absence of Sec13; moreover, a Sec31 mutant that lacked the flexible

\title{
Escaping normal tissue constraints in cancer
}

Tumorigenesis involves the clonal growth of single cells harbouring oncogenic alterations within a normal tissue. Leung and Brugge reveal the mechanisms that allow such cells to escape the growth constraints imposed by the tissue environment (Nature 482, 410-413; 2012).

The authors employed three-dimensional organotypic cultures, in which cells are organized in acini consisting of an epithelial layer surrounding a hollow lumen, to analyse the effects of oncogene expression on the outgrowth of single cells. They showed that in contrast to other oncogenic alterations, including expression of constitutively active AKT1 kinase, overexpression of the ERBB2 receptor tyrosine kinase in single cells drove their clonal outgrowth inside the lumen. Time-lapse imaging revealed that ERBB2-expressing cells translocated from the epithelial layer and into the lumen independently of their proliferative capacity. However, luminal extrusion, dependent on MAPK (mitogen-activated protein kinase) and PI(3)K (phosphatidylinositol-3-kinase) signalling downstream of ERBB2, was required for cell proliferation. MAPK-dependent activation of the ETS1 transcription factor promoted extrusion by inducing matrix metalloprotease expression for local remodelling of the epithelial environment. Concomitant overexpression of matrix metalloproteases and constitutively active AKT1 also allowed cell translocation and outgrowth. These findings underscore the importance of evading the suppressive influence of organized epithelial environments for cells containing oncogenic alterations to unleash their survival and expansion potential.

hinge region could compensate for Sec13 loss in vivo. These results suggest that $\operatorname{Sec} 13$ provides rigidity to the COPII coat to accommodate asymmetrically distributed cargo, and give insight into how coat proteins adapt to incorporate the wide variety of secretory proteins.

EJC

\section{Sensing microtubule dynamics}

Correct chromosome segregation depends on mechanisms sensing the attachment of chromosomes to the spindle. Lack of tension between sister kinetochores produces a conformational change in the kinetochore, but a detailed mechanistic understanding of this process is lacking. By visualizing GFP-tagged proteins in budding yeast, Bloom and colleagues now describe how reduced microtubule dynamics alters kinetochore and pericentric chromatin geometry (Curr. Biol. 22, 1-11; 2012)

The authors find that reducing microtubule dynamics with low concentrations of benomyl leads to a contraction of inner kinetochore height. Visualizing cohesin, a chromatinassociated protein complex, reveals that the cohesin barrel instead expands in response to benomyl. Using mutant strains, the authors find that both inner kinetochore contraction and cohesin expansion require Shugoshin 1 (Sgol, a protein that protects cohesin from degradation), Bubl kinase (known to recruit Sgol) and Bub1-mediated phosphorylation of histone $\mathrm{H} 2 \mathrm{~A}$, but not the spindle assembly checkpoint. The pericentric chromatin itself also expands following benomyl treatment, as observed by imaging centromere-tagged lacO arrays, and this is associated with suppressed chromatin dynamics. The authors suggest that the physical expansion of pericentric chromatin and cohesin exerts a force on the inner kinetochore, causing its contraction. Statistical maps of Bub1 and Sgo1 distribution further reveal that benomyl increases Sgo1 recruitment to the pericentric region and alters Bub1 distribution. Thus, Bub1 and Sgol are involved in translating the response to altered microtubule dynamics into geometrical changes in pericentric chromatin and cohesin structure.

CKR

By Emily J. Chenette, Christina Karlsson Rosenthal, Nathalie Le Bot and Alexia-Ileana Zaromytidou 